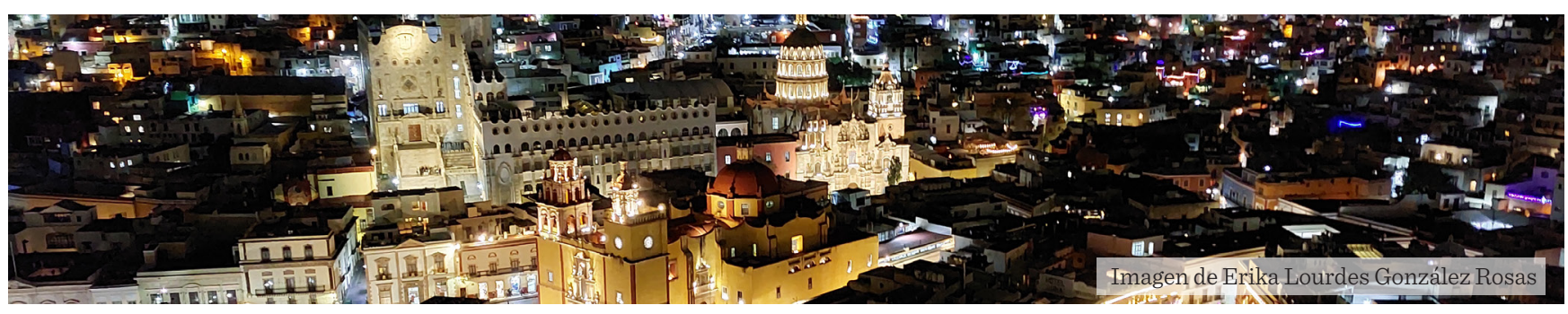

\title{
Efectos de la experiencia cultural en la percepción de excelencia e impacto económico de los asistentes al Festival Internacional Cervantino
} Effects of cultural experience on the perception of excellence and
economic impact of the attendees at the International Cervantino Festival

\author{
Erika Lourdes González-Rosas*, María de Lourdes Cárcamo-Solis² ${ }^{2}$ Ramón Navarrete-Reynoso ${ }^{3}$
}

\section{RESUMEN}

El Festival Internacional Cervantino (FIC) es el evento turístico cultural más importante de la ciudad de Guanajuato, que atrae cada año a miles de visitantes. El objetivo de esta investigación fue determinar los factores que forman la experiencia de los asistentes al FIC, así como su relación con la percepción de la excelencia y del impacto económico, mediante la adaptación de un modelo estructural teórico basado en el modelo escandinavo de "Skot-Hansen y Linko y Silvanto". Se empleó una metodología cuantitativa, sustentada en un modelo de ecuaciones estructurales, basado en una encuesta hecha de manera no aleatoria por conveniencia a 709 visitantes en 2017. Los resultados identificaron que los factores ilustración, empoderamiento y entretenimiento, provocaron en los asistentes del festival, una experiencia memorable que anima, profundiza y enaltece de forma significativa sus vidas, aspecto que no siempre se ha valorado en este tipo de actividades. Además, la experiencia influye en la excelencia apreciada, impactando en la percepción de la calidad y profesionalismo de la organización y el nivel artístico del FIC. Los asistentes de este festival también percibieron que la excelencia apreciada en los factores de ilustración, empoderamiento y entretenimiento, beneficia la imagen positiva de Guanajuato; al fortalecerla como destino turístico, lo que repercute económicamente. Se concluye que el FIC logra buenas prácticas y políticas culturales, lo que es arduo de alcanzar y puede ser un argumento a favor de su propuesta.

PALABRAS CLAVE: experiencia, excelencia, turismo, festival cultural.

\section{ABSTRACT}

The International Cervantino Festival (FIC) is the most important tourist cultural event in the city of Guanajuato since it attracts thousands of visitors every year. The objective of this research was to determine the factors that constitute the experience of FIC's attendees' as well as its relationship towards the perception of excellence and economic impact. A structural equation theoretical model based on the Scandinavian "Skot-Hansen and Linko and Silvanto" model was adapted for that purpose. A quantitative methodology was used, supported by a structural equations model, based on a survey with a non-random convenience sample of 709 visitors in 2017. Results identified that factors such as illustration, empowerment and entertainment produced a memorable experience among FIC attendees that encourages, deepens and heightens their lives in a meaningful way, an aspect that had not always been valued in these kinds of activities. Additionally, the experience also has an impact on perceived excellence, which impacts the perception of FIC's quality, professionalism, organization and artistic level. FIC's attendees also perceived that the excellence within illustration, empowerment and entertainment benefit Guanajuato's positive image strengthening the city as a touristic destination that leads to positive economic repercussions. It is concluded that FIC achieves good practices and cultural policies, a fact that is difficult to achieve and that can be an argument in favor of its cultural proposal.

KEYWORDS: experience, excellence, tourism, cultural festival.

\footnotetext{
*Correspondencia: gonzalez@ugto.mx/ Fecha de recepción: 16 de noviembre de 2020/Fecha de aceptación: 5 de noviembre de 2021/Fecha de publicación: 28 de enero de 2022.

${ }^{1}$ Universidad de Guanajuato, División de Ciencias Económico Administrativas, Departamento de Gestión y Dirección de Empresas, fraccionamiento El Establo s/n, Guanajuato, Guanajuato, México, C. P. 36250. ${ }^{2}$ Instituto Tecnológico de Morelia, Área de contaduría e ingeniería industrial, Morelia Michoacán, México. ${ }^{3}$ Universidad de Guanajuato, Departamento de Estudios Organizacionales, División de Ciencias Económico Administrativas, Guanajuato, Guanajuato, México.
} 


\section{INTRODUCCIÓN}

w La Organización de las Naciones Unidas para la Educación, la Ciencia y la Cultura (UNESCO, 2013) ha enfatizado la necesidad de analizar los resultados de las actividades creativas y culturales en aspectos económicos, ambientales, sociales y culturales. En ese contexto, el Festival Internacional Cervantino (FIC) se realiza cada año en el mes de octubre en la ciudad de Guanajuato, México. Es un evento suigéneris, porque combina las bellas artes con la cultura popular. Consta de actividades gratuitas y con costo en múltiples recintos. Generalmente, toca las temáticas de un país extranjero invitado y de un estado de México en específico (FIC, 2018). De acuerdo con la clasificación de Andersson y Getz (2009), el FIC se ubica en la categoría de público, lo que es importante en términos de costos, estructura, patrocinadores, voluntariado y gobernanza. Su versión XLV, en 2017, recibió 66000 turistas alojados en hoteles (Secretaría de Turismo, 2017). Mientras que en 2019 se hospedaron 64581 personas y se registró un total de 150281 asistentes reportándose una derrama económica de \$271.4 millones de pesos, de acuerdo con los datos del comité organizador (OTEG, 2019).

El FIC ha sido analizado desde las afectaciones al patrimonio, requerimientos de infraestructura y servicios (Barrera-Fernández y HernándezEscampa, 2017), experiencia según la afección, cognición, activismo, novedad y en comparación con otros festivales del mundo (Richards y RuizLanuza, 2017), también, respecto a la percepción de la experiencia (González-Rosas y GuevaraSanginés, 2017).

Desde hace tiempo, las acciones de este tipo se han visto como una forma de impulsar la imagen de las ciudades; en ocasiones, con la finalidad de afianzarse como sitios culturales (Richards y Wilson, 2004). En ese sentido, son una estrategia consolidada en la promoción de los destinos turísticos; además, sus servicios son intangibles, por tratarse de arte y cultura (Yeoman y col., 2004). Por esa razón, su estudio tiene diferentes perspectivas y es multivariado, va desde la antropología hasta la geografía, pa- sando por la economía y la religión, sin dejar de lado el turismo y el ocio (Getz, 2008). El campo de investigación incluye la segmentación (Kruger y col., 2019), la sustentabilidad (Mair y Jago, 2010) y la experiencia (Richards, 2019). Esta última, con la finalidad de identificar los factores que la motivan, mencionándose el ambiente, el escape, la conveniencia, calidad de la comida, facilidad y el staff, elementos relacionados con la satisfacción, intención de repetición y la promoción de los festivales culturales (Beckman y col., 2020). Existen antecedentes de un ambiente y/o cierto tipo de vivencias agradables que impulsan a la repetición de la visita (Mason y Paggiaro, 2012).

El estudio de los festivales abarca diferentes perspectivas, como el riesgo que implica tomar la decisión de asistir o no, lo cual se ha relacionado con la evaluación, satisfacción e intención del comportamiento. A mayor riesgo, impresión más negativa (Sohn y col., 2016). También, los aspectos de escape y la socialización afectan la percepción del entretenimiento, que se basa en el desempeño y la satisfacción o lealtad, como se demostró en el Festival de Blues Efes Pilsen de Turquía (Özdemir-Bayrak, 2011). Adicionalmente, la motivación ha sido abordada constantemente, atendiendo a la satisfacción y la lealtad, así como de diferencias entre los locales y los turistas (BáezMontenegro y Devesa-Fernández, 2017), o con el afecto, la cognición y actitudes de compartimiento entre primeros asistentes y repetidores, encontrando diferencias en el Festival de los Cerezos en Washington D.C. (Li y col., 2018). Mientras que el aspecto cocreativo ha detectado relación con la satisfacción y apego al lugar en festivales chinos (Zhang y col., 2019).

Moss y col. (2019) relacionaron la experiencia con factores como los procesos, el diseño del evento, el impacto personal, autenticidad y leveraing strategies (estrategias de apalancamiento) y con enfoque más psicológico o fenomenológico. Otros aspectos que se han analizado son la calidad, la diversidad, los grupos de interés, incrementalidad, simultaneidad, flexibilidad y enlace, factores que contribuyen 
a la satisfacción y la intención del comportamiento; así como la programación (Qi y col., 2012). La calidad es un factor tan importante para la experiencia que está relacionada con la intención o repetición de visita en términos de tráfico, seguridad, cultura, socialización, infraestructura y servicios (Pivac y col., 2019). Además, la experiencia influye en la satisfacción, lealtad, excelencia y tipo de asistente, marcando diferencias (Lee y col., 2017). Igualmente, existe evidencia de la correspondencia entre el ambiente y la satisfacción, el grupo, la intención de re-visita desde el aspecto de la teoría de la conducta (Hyungsuk y col., 2016).

Como ya se mencionó, un aspecto que destaca es la experiencia, y sus constructos teóricos han sido estudiados también, aunque en menor grado y de forma marginal en el contexto latinoamericano. Pine y col. (1998) fueron de los primeros autores en posicionarla como un factor determinante en el turismo $\mathrm{y}$, en lo específico, de los festivales, y establecieron el concepto de "economía de la experiencia", que se refiere al efecto en la industria turística. Es un tema que ha sido abordado ampliamente en los últimos años por Richards (2019) en diferentes entornos y contextos, incluidos los festivales. La experiencia turística se orienta hacia un mercado de creación de valor y, según la escuela escandinava une las actividades culturales con una expectativa de retorno y desarrollo económico (Bille, 2012). Además, es una de las formas de satisfacción y calidad de vida en los entornos turísticos (Kruger y col., 2013).

La literatura ha encontrado evidencia de modelos conceptuales sobre la experiencia en los festivales. Las vivencias emocionales y de comportamiento se han detectado como factores importantes en la necesidad de mejorar la experiencia de los visitantes a festivales de arte (Chen y col., 2018). Por otro lado, en festivales de comida y vino se ha evidenciado, que se fundamenta en un proceso co-creativo entre el asistente y los gestores, combinando el aprendizaje, el entretenimiento, el consumo y la autenticidad (la cultura, el patrimonio, la estética y la creatividad) (Getz, 2019).
Todo lo anterior debe contribuir a la percepción del impacto económico en la localidad sede y en la región, de lo contrario, se puede perder el balance de una adecuada gestión y buenas prácticas según la política cultural (Skot-Hansen, 2005). En este sentido, al ser el FIC un evento tanto cultural como popular, es importante evaluarlo buscando comprobar qué clase de experiencias genera en los asistentes y qué factores son importantes para ello.

El objetivo de esta investigación fue establecer los factores que conforman la experiencia de los asistentes al FIC, así como su relación con la percepción de la experiencia y del impacto económico en la ciudad de Guanajuato, México, mediante la adaptación de un cuestionario para comprobar empíricamente la validez de un modelo estructural teórico, basado en el escandinavo de Linko y Silvanto de las "6 E's", que tiene como precursor el Skot-Hansen de las "4 E's", integrando la parte cognitiva y la recreación, pero con la novedad de la excelencia.

\section{MATERIALES Y MÉTODOS}

Esta investigación está basada en el modelo de Skot-Hansen (2005), que describe el desarrollo histórico de las políticas y estructuras culturales urbanas, así como el debate sobre cómo comprender el nuevo papel de estas políticas en los entornos urbanos en el siglo XXI. Según este autor, la experiencia se basa en la estética y las emociones vividas, y la excelencia, con la calidad de los espectáculos y la ejecución (Skot-Hansen, 2002; Silvanto y col., 2008; Jochumsen y col., 2012; Olsen, 2013; Jochumsen y col., 2017). El modelo Skot-Hansen (2005) ofrece una herramienta útil para estudiar las razones fundamentales de las políticas culturales, y una propuesta para analizar los resultados que generan, buscando que las diferentes dimensiones no estén desequilibradas, ya que no es favorecedor, ni para el público o la sociedad, ni para los gestores, por ejemplo, que un proyecto cultural sólo genere resultados económicos pero que no fomente el fortalecimiento de los individuos. También se ha detectado, que su modelo es útil para analizar los argumentos, que usan los residentes para explicar por qué consideran importantes ciertas actividades (Linko y Silvanto, 2011). 
De acuerdo con Skot-Hansen (2005), existen cuatro fundamentos que han dominado los discursos de política cultural. Él los llama las 4 E's. Representan los acrónimos de los conceptos: ilustración (enlightenment), empoderamiento (empowerment), entretenimiento (entertainment) e impacto económico (economic impact). Este modelo analiza la parte cultural a la vez que lo conjuga con la diversión y el ocio, sin dejar de lado la excelencia. Ha sido utilizado en diferentes entornos y contextos, encontrando evidencia de que la cultura favorece la generación de experiencias (Skot-Hansen, 2002; Silvanto y col., 2008; Jochumsen y col., 2012; Olsen, 2013; Jochumsen y col., 2017).

En cuanto a la ilustración, se refiere a que las artes sirven para aumentar la percepción de aprendizaje, apreciación, educación y conocimiento, en tanto que la cultura y su patrimonio fortalecen el desarrollo democrático en la sociedad y la reflexión en los individuos. Este discurso está estrechamente relacionado con la idea de la "democratización de la cultura", que dominó las discusiones sobre políticas al respecto en la década de 1970, y destaca la importancia de ofrecer este tipo de actividades a todas las personas, independientemente de su origen social o ubicación geográfica. $\mathrm{Al}$ mismo tiempo, se discutió la "democracia cultural", que enfatiza el estatus igualitario de todas las formas de cultura (Silvanto y col., 2008; Linko y Silvanto, 2011).

El empoderamiento se refiere a la identidad, diversidad y cohesión social; igualmente, la idea de "democracia cultural" está interconectada con la lógica de este concepto, siendo el argumento central que debe usarse como un medio para empoderar a los ciudadanos, especialmente a aquellos que por una razón u otra están excluidos de la comunidad. Imagen e identidad local, cohesión e inclusión social y diversidad cultural son palabras clave en el discurso del empoderamiento (Skot-Hansen, 2005; Silvanto y col., 2008; Linko y Silvanto, 2011).

Por su parte, el concepto de entretenimiento está relacionado con el valor de que la cultura debería estar asociada con el ocio y la recreación, la necesidad de jugar y relajarse (SkotHansen, 2005). La noción de una "ciudad divertida", un mundo lleno de actividades y entretenimientos espectaculares (Zukin, 1995) donde la gente puede disfrutar es central en este discurso (Linko y Silvanto, 2011), lo que también ha sido abordado por Richards (2019).

Es posible que los asistentes no estén buscando conscientemente la ilustración o el empoderamiento, pero ciertamente buscan experiencias interesantes, la quinta E. Esto significa que, cuando realmente se vive el arte también se obtiene una nueva percepción y/o se siente una conexión social con quienes participan en el mismo evento, con el arte y con la cultura, lo que también se ha llamado una vivencia estética, que anima y profundiza en la existencia de las personas (Skot-Hansen, 2005; Silvanto y col., 2008; Linko y Silvanto, 2011).

Linko y Silvanto (2011) resaltan la importancia de la calidad artística como un factor necesario tanto para los responsables de las políticas culturales como para las intervenciones artísticas. Por lo tanto, agregaron una sexta E, Excelencia (Excellence) al modelo Skot-Hansen. Este elemento surgió como resultado del análisis de los datos empíricos y de la discusión sobre política cultural en la que la democratización de la cultura coincidió con las demandas de excelencia. Además de estas fuentes, los medios de comunicación revisan activamente el desempeño y nivel artístico de las representaciones. Esto se aplica a los artistas intérpretes o ejecutantes individuales, así como a la organización general (Linko y Silvanto, 2011). En la gestión de la calidad, la excelencia es un sinónimo de tener lo mejor o el más alto estándar en la interpretación y un sentido de diferenciación.

La literatura muestra que se ha encontrado evidencia sobre cómo los eventos culturales y artísticos influyen en la apreciación del impacto económico que tienen los asistentes a los lugares donde se diseñan y ejecutan estas actividades (Skot-Hansen, 2005). Es cómo estos generan resultados económicos en la ciudad, imagen, em- 
pleos, turismo y contribuyen al desarrollo local, regional y urbano.

Finalmente Skot-Hansen (2005), señala que todos estos fundamentos son instrumentales de una forma $u$ otra de la experiencia cultural y afirma, que se debería prestar más atención a su análisis en la política sobre el tema. Por esta razón, la presente investigación buscó comprobar: primero, si los asistentes al FIC aprenden, conocen arte, se sienten incluidos, obtienen bienestar, se distraen; segundo: si esos elementos, que son dimensiones de la experiencia, son determinantes para la excelencia percibida; es decir, si favorecen una mejor impresión de la organización y del nivel artístico de sus presentaciones; tercero: cómo la excelencia vivida en el FIC puede influir en la apreciación del impacto económico de la ciudad como un aspecto positivo para Guanajuato. Este punto es importante porque no siempre se analiza la percepción de los eventos y sus beneficios o perjuicios.

\section{Instrumento}

Se utilizó el "Cuestionario de Opinión Sobre el Festival Internacional Cervantino" diseñado por las autoras González-Rosas y Guevara-Sanginés en 2015, mejorado en la versión 2016 del FIC (González Rosas y Guevara-Sanginés, 2017) y ajustado en sus escalas para su aplicación en 2017 , resultando con 43 reactivos. Una vez analizadas las pruebas de consistencia interna del piloteo, se decidió aprobarlo para su aplicación en el presente estudio.

\section{Métodos}

El método fue cuantitativo, correlacional y multivariado. Se utilizó una encuesta para determinar, con aspectos sociodemográficos, el perfil del participante y medir las escalas de ilustración, empoderamiento, entretenimiento, impacto económico, experiencia y excelencia (SkotHansen, 2005; Linko y Silvanto, 2011) en cuanto a lo específico del FIC. El tipo de muestreo fue no probabilístico intencional. El estudio se realizó en la versión XLV del FIC en sus 19 d de duración, del 11 al 29 de octubre de 2017. De los 66000 visitantes, se aplicaron 709 encuestas, en dos recintos ubicados en la capital de Guanajuato, México. Participó un equipo de 10 encuestadores previamente capacitados, quienes entregaron el cuestionario para ser contestado a cada una de las personas que salían de las presentaciones, sin intervenir en sus respuestas, por lo que también fue observacional. Posteriormente, la información fue sistematizada y capturada en google drive y en paquete estadístico para ciencias sociales (SPSS, por sus siglas en inglés: Statistical Package for the Social Sciences) (SPSS, 2016) versión 24, para trabajar el Análisis Factorial Exploratorio, y luego copiada a Excel para ser procesada en Mínimos Cuadrados Parciales (SmartPLS, por sus siglas en inglés: Partial Least Squares) (SmartPLS, 2015) versión 3.0, con la finalidad de construir las relaciones de causalidad entre los constructos y poder obtener el Análisis Factorial Confirmatorio y finalmente, el Modelo de Ecuaciones Estructurales (SEM, por sus siglas en inglés: Structural Equation Modeling).

Con el propósito de precisar la calidad del modelo estructural se siguieron los siguientes pasos: Primero se evaluó la multicolinealidad, a través de la Fiabilidad Compuesta (FC) según el valor crítico < 5.0 y > 0.20 (Hair y col., 2017). Segundo, para los coeficientes de determinación $\mathrm{R}^{2}$ se utilizaron los rangos de Chin (1998) $\mathrm{R}^{2}$ : $>0.20$ = Débil; $>0.33$ = Moderada; $\mathrm{y}>0.67=$ sustancial. Tercero, se midió la relevancia predictiva de los constructos dependientes de acuerdo con los valores de $Q^{2}$ : > 0 (Geisser, 1974; Stone, 1974). Esto se obtiene al realizar la prueba Blindfolding en el SmartPLS, que examina qué tan bien se podría predecir una parte sistemática y supuesta de los datos perdidos mediante los parámetros estimados por el algoritmo PLS y el modelo de ecuaciones estructurales. Cuarto, de acuerdo con los resultados, se valoró la significancia de las relaciones planteadas del modelo estructural, para continuar. Quinto, se evaluó el impacto relativo a través del efecto de la medida $\mathrm{F}^{2}$ con base en los siguientes coeficientes: $\mathrm{F}^{2}>0.02$ = pequeño; $>0.15=$ mediano; > 0.35 = grande (Cohen, 1988).

Adicionalmente, se calculó la fiabilidad de las escalas, el alpha de Cronbach con base en los es- 
tándares de Nunnally y Bernstein (1994) > 0.7, la FC > 0.7 (Hair y col., 2017) y la Varianza Extraída Promedio (AVE, por sus siglas en inglés: Average Varience Extracted) superior a 0.5 (Fornell y Larcker, 1981).

Finalmente, para la comprobación del modelo se aplicó la técnica de Bootstrapping, con el objeto de identificar la significancia de las cargas factoriales de acuerdo con los coeficientes Path, considerando los valores de t > 1.96 ó 2.57 y $\mathrm{P}<0.5 \mathrm{y}<0.1$, de Hair y col. (2017).

Con respecto a los ítems pertenecientes al constructo de la ilustración, tienen que ver con el proceso de cognición de la cultura que promueve el festival. Se hicieron preguntas como "el FIC incrementó mi cultura, me generó interés de conocer el país o estado invitado". En cuanto al empoderamiento se incluyeron ítems relacionados con "la identidad y unión con Guanajuato, impacto en visitantes y locales". Para el constructo entretenimiento, se basó en preguntas como "el FIC me llenó de bienestar, fue una buena distracción y fue divertido". En la experiencia, las preguntas se basan en "si el evento del FIC me ha generado sentimientos de armonía, si ha sido una buena experiencia y como una manera de conocer el arte". En la excelencia están incluidos los ítems asociados con "la calidad artística, el profesionalismo de los organizadores y el nivel de los artistas”. Mientras que el impacto económico se re- fiere a "la percepción del número de empleos creados, el fomento de la infraestructura y servicios en Guanajuato, impulsó a los servicios turísticos relacionados como fuente de desarrollo económico para las familias guanajuatenses".

\section{RESULTADOS Y DISCUSIÓN}

El tamaño de todas las cargas factoriales de los constructos se ubicó en un rango entre los valores 0.648 y 0.822 , superior a la recomendación (> 0.6) de Bagozzi y Yi (1988). Los datos demostraron una alta consistencia interna en los constructos en cada caso. El alpha de Cronbach sobrepasó el valor de 0.70, de acuerdo con los estándares de Nunnally y Bernstein (1994). La FC se presentó arriba del valor crítico de 0.7, considerado como bueno (Hair y col., 2017). La AVE resultó superior a 0.50 (Fornell y Larcker, 1981) en todos los constructos, por lo que se pudo comprobar que el SEM es robusto, altamente válido y confiable (Tabla 1).

Los resultados sociodemográficos de la muestra se exponen en la Tabla 2 , donde se reporta una mayor participación de mujeres (16\% más que hombres), con un promedio general de edad de 27 años (DE = 11.31); respecto a la ocupación, casi el $60 \%$ son estudiantes, seguido de profesionistas (18.5\%), mientras que en el nivel educativo se observa un alto porcentaje con licenciatura o posgrado, casi el $80 \%$, lo que denota que el conjunto tiene un sesgo a ambas variables. Los datos reflejan que los asistentes

Tabla 1. Consistencia Interna y Validez Convergente del instrumento.

Table 1. Internal Consistency and Convergent Reliability of the Instrument.

\begin{tabular}{|l|c|c|c|c|}
\hline \multicolumn{4}{|c|}{ Validez Convergente } & Consistencia Interna \\
\hline \multicolumn{1}{|c|}{ Constructo } & $\begin{array}{c}\text { Rangos } \\
\text { de Cargas } \\
\text { Factoriales de } \\
\text { indicadores }\end{array}$ & $\begin{array}{c}\text { AVE (Varianza } \\
\text { Extraída } \\
\text { Promedio) } \\
>0.5\end{array}$ & $\begin{array}{c}\text { FC (Fiabilidad } \\
\text { Compuesta) } \\
>0.7\end{array}$ & $\begin{array}{c}\text { Alfa de Cronbach } \\
>0.7\end{array}$ \\
\hline Ilustración & 0.683 a 0.806 & 0.561 & 0.899 & 0.869 \\
\hline Empoderamiento & 0.648 a 0.797 & 0.573 & 0.869 & 0.812 \\
\hline Entretenimiento & 0.667 a 0.801 & 0.560 & 0.910 & 0.887 \\
\hline Experiencia & 0.715 a 0.787 & 0.564 & 0.866 & 0.806 \\
\hline Excelencia & 0.669 a 0.786 & 0.544 & 0.905 & 0.875 \\
\hline Impacto Económico & 0.735 a 0.822 & 0.614 & 0.905 & 0.875 \\
\hline
\end{tabular}


Tabla 2. Distribución sociodemográfica de la muestra.

Table 2. Sociodemographic sample distribution.

\begin{tabular}{|l|c|}
\hline \multicolumn{1}{|c|}{ Atributos } & (\%) \\
\hline \multicolumn{1}{|c|}{ Género } & 58 \\
\hline Femenino & 42 \\
\hline Masculino & \\
\hline \multicolumn{1}{|c|}{ Estudiante } \\
\hline Profesionista & 59.8 \\
\hline Personal de oficina & 18.5 \\
\hline Artesano & 9.1 \\
\hline Ama de casa & 3.8 \\
\hline Comerciante y ventas & 3.4 \\
\hline Jubilados & 3.2 \\
\hline Gerentes & 1.1 \\
\hline \multicolumn{1}{|c|}{ Procedencia } & 1.0 \\
\hline Estado de Guanajuato & 83.4 \\
\hline Resto de México & 7.2 \\
\hline Ciudad de México & 6.3 \\
\hline Guadalajara & 2.2 \\
\hline Estados Unidos de América & 0.5 \\
\hline Canadá & 0.2 \\
\hline Otros & 0.2 \\
\hline \multicolumn{1}{|c|}{ Nivel educativo } & \\
\hline Primaria o menos & 0.7 \\
\hline Secundaria & 0.9 \\
\hline Preparatoria & 17.8 \\
\hline Técnico & 1.6 \\
\hline Licenciatura & Eños \\
\hline Posgrado & \\
\hline \multicolumn{1}{|c|}{ Edad promedio } & $11.31 \mathrm{DE}$ \\
\hline Edad & \\
\hline & \\
\hline
\end{tabular}

$\mathrm{DE}=$ desviación estándar.

al FIC tienen una baja representatividad para otros grupos según la procedencia, ya que el $8.5 \%$ eran foráneos de dos metrópolis cercanas a Guanajuato (Ciudad de México y Guadalajara) y casi $1 \%$ eran extranjeros (Estados Unidos de América y Canadá, principalmente) aunque a estos no se les preguntó si habían viajado expresamente al evento o aprovecharon su estadía en el país para atender al FIC.

Otro criterio para establecer la calidad del modelo estructural es la prueba Stone-Geisser de relevancia predictiva $Q^{2}$ (Geisser, 1974; Stone, 1974). Para el modelo estructural, el empoderamiento presentó una relevancia predictiva $\mathrm{Q}^{2}=0.297$, el entretenimiento un $\mathrm{Q}^{2}=0.306$, la ilustración $\mathrm{Q}^{2}=0.249$, la excelencia un $\mathrm{Q}^{2}=0.123$ y el impacto económico $\mathrm{Q}^{2}=0.110$, por lo que se asume que estos constructos tienen un importante valor predictivo, ya que su $\mathrm{Q}^{2}>0$ (Hair y col., 2017). Adicionalmente, se calculó la prueba $\mathrm{F}^{2}$ (Cohen, 1988) para proporcionar información acerca de la magnitud del efecto (Tabla 3), el cual es grande en el caso de entretenimiento-experiencia (1.254), y empoderamiento-experiencia (1.10) mientras que es medio en los vínculos de ilustración-experiencia (0.83), experienciaexcelencia (0.303) y excelencia-impacto económico (0.231). El valor predictivo es la capacidad con la que estas relaciones originan un efecto multiplicativo, siempre y cuando se vean incrementadas en sus valores para producir buenos resultados en la atracción de más turistas que gusten de la experiencia del FIC, generando buenas expectativas para que los organizadores inviertan más recursos que se traduzcan no sólo en la visita repetida, sino sean un reflejo de la percepción del desarrollo económico a través del aumento en el empleo, en el consumo de bienes y servicios de la industria turística y, por tanto, impacte en la apreciación de Guanajuato como un buen destino turístico que visitar.

El algoritmo PLS mostró un excelente ajuste incremental del modelo, con valor de 0.80, mientras que los resultados del modelado se muestran en la Figura 1. Se puede observar que el modelo estructural tiene calidad explicativa, al analizar sus respectivos coeficientes de determinación $\mathrm{R}^{2}$ de sus variables como la ilustración (45.4\%), el empoderamiento (52.3\%) y el entretenimiento (55.6\%), que muestran que la experiencia cultural se explica de forma moderada por estas tres variables tangibles. Esta, a su vez, explica débil, en un $23.2 \%$ a la excelencia y al impacto económico con un $18.6 \%$. En cuanto a los coeficientes estandarizados $\beta$, la experiencia cultural se relaciona con el entretenimiento en 0.746, con el empoderamiento en 0.724 y con la ilustración en 0.674. Ade- 
Tabla 3. Resultados del modelo estructural (PLS SEM).

Table 3. Structural Equation Model Results (PLS SEIM).

\begin{tabular}{|l|c|c|c|c|c|c|}
\hline Hipótesis & Path & $\begin{array}{c}\text { Coeficiente } \\
\text { Estandarizado } \beta\end{array}$ & T-valor & P -valor & $\mathbf{F}^{2}$ & Decisión \\
\hline H1 & $\begin{array}{c}\text { Ilustración--- } \\
\text { Experiencia }\end{array}$ & $0.674^{*}$ & 27.100 & 0.000 & 0.830 & $\begin{array}{c}\text { Fuertemente } \\
\text { soportada }\end{array}$ \\
\hline H2 & $\begin{array}{c}\text { Empoderamiento--- } \\
\text { Experiencia }\end{array}$ & $0.724^{*}$ & 34.970 & 0.000 & 1.100 & $\begin{array}{c}\text { Fuertemente } \\
\text { soportada }\end{array}$ \\
\hline H3 & $\begin{array}{c}\text { Entretenimiento--- } \\
\text { Experiencia }\end{array}$ & $0.746^{* *}$ & 33.049 & 0.000 & 1.254 & $\begin{array}{c}\text { Fuertemente } \\
\text { soportada }\end{array}$ \\
\hline Experiencia--- & $0.482^{* *}$ & 12.931 & 0.000 & 0.303 & $\begin{array}{c}\text { Fuertemente } \\
\text { soportada }\end{array}$ \\
\hline Excelencia & $\begin{array}{c}\text { Excelencia--- } \\
\text { Impacto Económico }\end{array}$ & $0.433^{* *}$ & 11.535 & 0.000 & 0.231 & $\begin{array}{c}\text { Fuertemente } \\
\text { soportada }\end{array}$ \\
\hline
\end{tabular}

Altamente significante: ${ }^{* *} \mathrm{P}<0.01$; Significante: ${ }^{*} \mathrm{P}<0.05$ y $\mathrm{P}<0.10$, ns $=$ No significante.

Tamaño del Efecto: $\mathrm{F}^{2}>0.02$ = Efecto Pequeño, $\mathrm{F}^{2}>0.15=$ Efecto Mediano; $\mathrm{F}^{2}>0.35=$ Efecto Grande

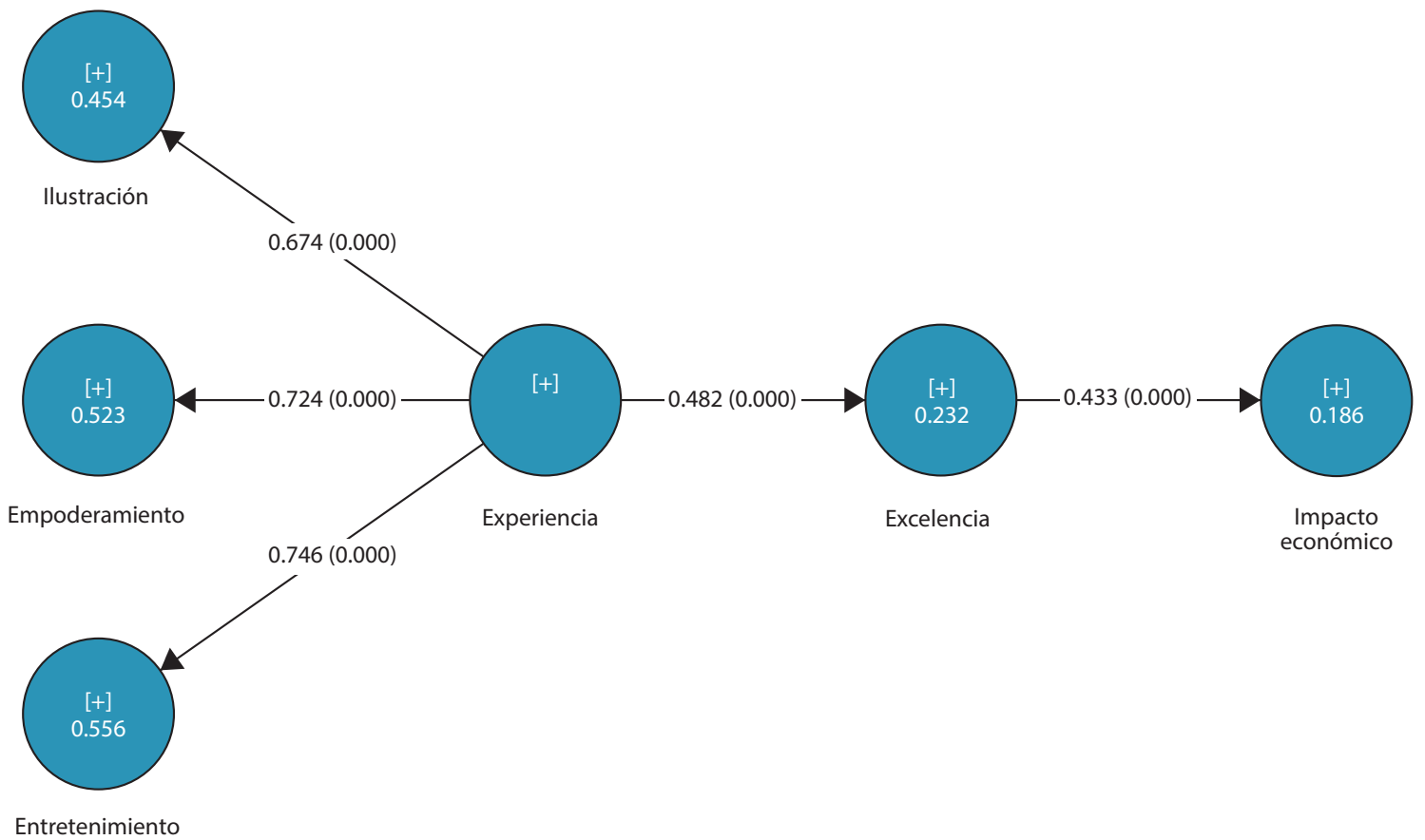

*Los valores dentro de los círculos corresponden al coeficiente de determinación $\mathrm{R}^{2}$.

- Figura 1. Modelo de Ecuaciones Estructurales (SEM).

Figure 1. Structural Equation Model (SEM).

más, se relaciona con la excelencia en 0.482 y la excelencia con el impacto económico en 0.433 (Figura 1 y Tabla 3). Por lo que estos registros dan evidencia de la fortaleza que tiene el modelado estructural para explicar las relaciones causales entre las variables de es- tudio de la presente investigación, definiendo como crítico este método de estudio, ya que señala las estrategias a seguir para mejorar la experiencia cultural del FIC y promover el turismo tanto nacional como internacional en Guanajuato. 
Es importante tomar en cuenta que el modelado de ecuaciones estructurales es un método de análisis de datos que ha sido ampliamente usado en algunos campos y tiene 3 ventajas: a) los errores de medición pueden ser controlados, b) la medición de variables puede ser fácilmente usada, c) es posible una evaluación estadística del modelado teórico. Es decir, como investigadores, se define qué tan bien se construye el modelo teórico ajustando los datos reales y aceptando el modelo como válido, o se modifica según sea necesario. Sin embargo, el modelado de ecuaciones estructurales es complicado (Kang y Ahn, 2021), ya que requiere de arduo trabajo estadístico para modelar una realidad, como es el caso de la experiencia cultural que representa el FIC para el turismo local, nacional e internacional. En este sentido, los modelos estructurales han sido utilizados para estudiar la industria turística mexicana (Herrera-Rodríguez y col., 2020) y, en específico, en las áreas naturales de México. Ávila-López y Pinkus-Rendón (2018) analizaron cómo lo económico influyó en la dimensión social con la experiencia, cohesión, compromiso, equidad, capital social, derechos humanos y educación.

En esa tesitura, los resultados de esta investigación, muestran la relación del constructo experiencia cultural del FIC con la ilustración, el empoderamiento y el entretenimiento, al mismo tiempo que con la excelencia, es decir, la calidad del servicio cultural, en este caso de los eventos artísticos, y en la percepción de los resultados económicos, mostrándose en la Figura 1. Los constructos se vieron fuertemente apoyados por los resultados de la aplicación de la prueba del Bootstrapping en el software SmartPLS, que confirmaron que las pruebas $\mathrm{T}$ y el valor de $\mathrm{P}$ establecen que las relaciones entre ellos fueron de naturaleza positiva y altamente significativa, aprobando, por tanto, dichas relaciones entre los constructos o variables de análisis (Tabla 3).

En este sentido, el modelo propuesto es holístico e integral y los resultados contribuyen al constructo de la experiencia, primero hacia el entretenimiento y el empoderamiento. Conforme a lo encontrado por Richards (2019), aborda el aspecto cognitivo, que, en lo general, no se considera especialmente para festivales a través del aprendizaje, conocimiento y educación, pero también la parte emotiva, al contemplar el entretenimiento, el escape y el ocio, tema que ha sido ampliamente estudiado (Özdemir-Bayrak, 2011; Getz, 2019; Heidari y col., 2019; Richards, 2019), pero poco en México. En lo específico del empoderamiento, los asistentes encuentran un sentido de identidad, inclusión, cohesión y diversidad, similar a lo descubierto por Crespi-Vallbona y Richards (2007). Derivado de los factores ilustración, entretenimiento y empoderamiento, los asistentes reflejan que viven una experiencia cultural nítida, estética y en general buena.

Con respecto a la excelencia, es impulsada por la experiencia, tema del que existen antecedentes (Pivac y col., 2019); es decir, parte de significar para la audiencia una buena experiencia requiere un nivel satisfactorio de excelencia en lo artístico y cultural. La excelencia se relaciona con el estándar más alto de calidad, en este caso, artística y de los servicios del festival. Determinar la calidad en los eventos es importante, porque se ha relacionado con la satisfacción y la intención de visita (Qi y col., 2012).

Finalmente, los resultados mostraron que la buena experiencia y la excelencia influyen en la percepción positiva del FIC para el fortalecimiento del destino turístico, imagen, empleo, capacitación y servicios turísticos de la ciudad de Guanajuato, lo que difícilmente se logra en los lugares donde los asistentes captan más bien lo negativo; sin embargo, en este estudio los resultados muestran que se responde al planteamiento de-Brito y Richards (2017), de que el evento sirve al destino.

Es trascendental que se hayan determinado los constructos en la experiencia del FIC, ya que se sabe de la importancia de que los sitios turísticos planeen y organicen sus actividades, incluso diseñen y desarrollen su portafolio, con la 
posibilidad de aumentar el número de visitantes y los beneficios (Getz y Page, 2016; Ziakas y Getz, 2021), en este caso, para la ciudad de Guanajuato y su principal suceso turístico y cultural.

\section{CONCLUSIONES}

La modelización de ecuaciones estructurales permite inferir que los factores de ilustración, empoderamiento y entretenimiento son un reflejo significativo del constructo experiencia, que impacta de manera significativa en la excelencia del Festival Internacional Cervantino (FIC). A su vez, esta incide significativamente en la percepción del beneficio económico en la ciudad de Guanajuato. La pesquisa de la experiencia es un tema concéntrico; en este caso, ayuda a determinar dónde el FIC debe po- sicionar sus tácticas, esfuerzos y recursos, ya que se concluye que no solo proporciona un servicio, también brinda una vivencia memorable para los que asisten y que no debe descuidarse su realización y propuesta artística. La planeación del FIC permite mejorar la experiencia de los visitantes e ir aumentando su número sin descuidar la calidad del evento. Finalmente, se puede mencionar como limitantes del estudio el tamaño de la muestra y que el modelo SEM sólo explica el caso del FIC. Como futuras líneas de investigación queda pendiente el análisis de la percepción de los residentes de la ciudad respecto al festival en aspectos más específicos, como su afectación al ambiente y la medición cuantitativa de los resultados económicos que acarrea.

\section{REFERENCIAS}

Andersson, T. D. and Getz, D. (2009). Festival Ownership. Differences between Public, Nonprofit and Private Festivals in Sweden. Scandinavian Journal of Hospitality \& Tourism. 9(2/3): 249-265.

Ávila-López, C. y Pinkus-Rendón, M. (2018). Teorías económico-ambientales y su vínculo con la dimensión social de la sustentabilidad en Áreas Naturales Protegidas. CienciaUAT.13(1): 108-122.

Báez-Montenegro, A. and Devesa-Fernández, M. (2017). Motivation, satisfaction and loyalty in the case of a film festival: differences between local and non-local participants. Journal of Cultural Economics. 41(2): 173-195.

Bagozzi, R. P. and Yi, Y. (1988). On the evaluation of structural equation models. Journal of the Academy of Marketing Science. 16(1): 74-94.

Barrera-Fernández, D. and Hernández-Escampa, M. (2017). Events and placemaking: the case of the Festival Internacional Cervantino in Guanajuato, Mexico. International Journal of Event and Festival Management. 8(1): 24-38.

Beckman, E., Shu, F., and Pan, T. (2020). The application of enduring involvement theory in the development of a success model for a craft beer and food festival. International Journal of Event and Festival Management. 11(4): 397-411.

Bille, T. (2012). The Scandinavian approach to the experience economy - does it make sense? International Journal of Cultural Policy. 18(1): 93-110.

Chen, Y. C., King, B., and Lee, H. W. (2018). Experiencing the destination brand: Behavioral intentions of arts festival tourists. Journal of Destination Marketing \& Management. 10: 61-6\%.

Chin, W. W. (1998). The partial least squares approach to structural equation modeling. In G. A. Marcoulides (Ed.), Modern Methods for Business Research (pp. 295-358). Mahwah: Lawrence Erlbaum.

Cohen, J. (1988). Statistical power analysis for the behavioral sciences. NJ: Lawrence Erlbaum, Mahwah. 66 Pp.

Crespi-Vallbona, M. and Richards, G. (2007). The Meaning of Cultural Festivals. International Journal of Cultural Policy. 13(1): 103-122.

De-Brito, M. P. and Richards, G. (2017). Events and placemaking. International Journal of Event and Festival Management. 8(1):2-7.

FIC, Festival Internacional Cervantino (2018). Festival Internacional Cervantino. [En línea]. Disponible en: http:// www.festivalcervantino.gob.mx. Fecha de consulta: 12 de noviembre de 2018.

Fornell, C. G. and Larcker, D. (1981). Evaluating structural equation models with unobservable variables and measurement error, Journal of Marketing Research. 18: 39-50.

Geisser, S. (1974). A predictive approach to the random effects model. Biometrika.61(1): 101-10\%.

Getz, D. (2008). Event tourism: Definition, evolution, and research. Tourism Management. 29(3): 403-428.

Getz, D. (2019). Wine and Food Events: Experiences and Impacts BT. In M. Sigala y R. N. S. Robinson (Eds.), Wine Tourism Destination Management and Marketing: Theory and Cases (pp. 143-164). Saint Lucia, PR: Springer International 
Publishing.

Getz, D. and Page, S. J. (2016). Progress and prospects for event tourism research. Tourism Management. 52: 593-631.

González-Rosas, E. y Guevara-Sanginés, M. (2017). Experiencia Cultural de los Eventos del Festival Internacional Cervantino. En Richards, G. y Lanuza, A. (Eds.), Experiencias Turísticas de Festivales y Eventos. [En línea]. Disponibleen: https://d1wqtxts1xzler.cloudfront.net/53383331/ PASOS_Edita_17.pdf?1496572192=\&response-content-dis position=inline $\% 3 \mathrm{~B}+$ filename\%3DExperiencias_turisticas _de_festivales_y.pdf\&Expires=160229326\&:Signature $=$ gj $\sim$ Rdup48NkYASK7gkz7C8kqiKfOJJhXE8mIzBW8osi3R6AkKgR WNj. Fecha de consulta: 12 de octubre de 2020.

Hair, J. F., Hult, G. T. M., Ringle, C. M., and Sarstedt, M. (2017). A primer on partial least squares structural equation modeling (PLS-SEM). (Second Edition). Thousand Oaks, CA: SAGE Publications. 137-190 Pp.

Heidari, K., Heydarinejad, S., Saffari, M., and Khatibi, A. (2019). Investigating the leisure behavior of Iranians: the structural model of serious leisure, recreation specialization and place attachment. Journal of Policy Research in Tourism, Leisure and Events. 13(1): 77-93.

Herrera-Rodríguez, M., Gutiérrez-Ortiz, E., Moreno-Herrera, A. y Segura-Zaleta, J. (2020). Factores de capital humano que impactan el capital social: estudio de hoteles del sur de Tamaulipas. CienciaUAT.15(1): 147-161.

Hyungsuk, C., Kwangho, A., and James, F. P. (2016). An integrated model of festival revisit intentions: Theory of planned behavior and festival quality/satisfaction. International Journal of Contemporary Hospitality Management. 28(4): 818-838.

Jochumsen, H., Skot-Hansen, D., and Hvenegaard-Rasmussen, C. (2012). The four spaces - a new model for the public library. New Library World. 113(11/12): 586-597.

Jochumsen, H., Skot-Hansen, D., and Hvenegaard-Rasmussen, C. (2017). Towards Culture 3.0 - performative space in the public library. International Journal of Cultural Policy. 23(4): 512-524.

Kang, H. and Ahn, J. W. (2021). Model setting and interpretation of results in research using structural equation modeling: A check list with guiding questions for reporting. Asia Nursing Research.1(6).

Kruger, M., Saayman, M., and Hull, J. (2019). A motivation-based typology for natural event attendees. Journal of Policy Research in Tourism, Leisure and Events. 11(1): 35-53.

Kruger, S., Rootenberg, C., and Ellis, S. (2013). Examining the Influence of the Wine Festival Experience on Tourists' Quality of Life. Social Indicators Research. 111(2): 435-452.

Lee, W., Sung, H., Suh, E., and Zhao, J. (2017). The effects of festival attendees' experiential values and satisfaction on re-visit intention to the destination. International Journal of Contemporary Hospitality Management. 29(3): 10051027.

Li, J., Deng, J., and Pierskalla, C. (2018). Impact of attendees' motivation and past experience on their attitudes toward the National Cherry Blossom Festival in Washington, D.C. Urban Forestry \& Urban Greening. 36: 57-67.

Linko, M. and Silvanto, S. (2011). Infected by Arts Festivals: Festival Policy and Audience Experiences in the Helsinki Metropolitan Area. Journal of Arts Management, Law \& Society. 41(4): 224-239.

Mair, J. and Jago, L. (2010). The development of a conceptual model of greening in the business events tourism sector. Journal of Sustainable Tourism. 18(1): 777-94.

Mason, M. C. and Paggiaro, A. (2012). Investigating the role of festival scape in culinary tourism: The case of food and wine events. Tourism Management. 33(6): 1329-1336.

Moss, J., Whalley, P. A., and Elsmore, I. (2019). Phenomenological psychology \& descriptive experience sampling: a new approach to exploring music festival experience. Journal of Policy Research in Tourism, Leisure and Events. 12(3): 382-400.

Nunnally, J. C. and Bernstein, I. H. (1994). Psychometric Theory (Third edition). New York: McGraw-Hill. 736 Pp.

Olsen, C. (2013). Re-thinking festivals: a comparative study of the integration/marginalization of arts festivals in the urban regimes of Manchester, Copenhagen and Vienna. International Journal of Cultural Policy. 19(4): 481-500.

OTEG, Observatorio Turístico del Estado de Guanajuato (2019). Perfil del visitante 2019. El 477 Festival Internacional Cervantino. [En línea]. Disponible en: http://www. observatorioturistico.org/publicaciones/ver_todos/7. Fecha de consulta: 19 de octubre de 2021.

Özdemir-Bayrak, G. (2011). Festival motivators and consequences: a case of Efes Pilsen Blues Festival, Turkey. Anatolia: An International Journal of Tourism \& Hospitality Research. 22(3): 378-389.

Pine, B., Joseph, I. I., and Gilmore, J. H. (1998). Welcome to the Experience Economy. Harvard Business Review. 76(4): 97-105.

Pivac, T., Blešić, I., Kovačić, S., Besermenji, S., and Lesjak, M. (2019). Visitors' Satisfaction, Perceived Quality, and Behavioral Intentions: The Case Study of Exit Festival. Journal of the Geographical Institute "Jovan Cvijic" SASA. 69(2): 123-134.

Qi, Y., Hanqin, Z., and Mimi, L. (2012). Programming quality of festivals: conceptualization, measurement, and relation to consequences. International Journal of 
Contemporary Hospitality Management. 24(4): 653-673.

Richards, G. (2019). Measuring the dimensions of event experiences: applying the Event Experience Scale to cultural events. Journal of Policy Research in Tourism, Leisure and Events. 12(3): 422-436.

Richards, G. y Ruiz-Lanuza, A. (2017). Experiencias Turísticas de Festivales y Eventos. [En línea]. Disponible en: https://dwqtxts1xzle7.cloudfront.net/53383331/PASOS_ Edita_17.pdf?1496572192=\&response-content-disposition =inline\%3B+filename\%3DExperiencias_turisticas_de_fes tivales_y.pdf\&Expires=1602293260\&Signature $=g j \sim$ Rdup48NkYASK7gkz7C8kqiKfOJJhXE8mIzBW8osi3R6AkKgRWNj. Fecha de consulta: 12 de octubre de 2020.

Richards, G. and Wilson, J. (2004). The impact of cultural events on city image: Rotterdam, cultural capital of Europe 2001. Urban Studies (Routledge). 41(10): 1931-1951.

Secretaría de Turismo (2017). Perfil del Visitante del Festival Internacional Cervantino. Gobierno del Estado de Guanajuato. Secretaría de Turismo, Observatorio Turístico. [En línea]. Disponible en: http://www.observatorioturistico. org/publicaciones/ver_todos/7. Fecha de consulta: 12 de noviembre de 2020.

Silvanto, S., Linko, M., and Cantell, T. (2008). From enlightenment to experience: cultural centres in Helsinki neighbourhoods. International Journal of Cultural Policy. 14(2): 165-178.

Skot-Hansen, D. (2002). Danish Cultural Policy--From Monoculture Towards Cultural Diversity. International Journal of Cultural Policy. 8(2): 197-210.

Skot-Hansen, D. (2005). Why urban cultural policies Danish National Research Database. In J. Robinson (Ed.), Eurocult21 Integrated Report (pp. 21-40). Helsinki, PR: EUROCULT21.

SmartPLS, Smart Partial Least Squares (2015). Versión 3.0. [En línea]. Disponible en: https://www.smartpls.com/. Fecha de consulta: 14 de julio de 2020.

Sohn, H. K., Lee, T. J., and Yoon, Y. S. (2016). Relationship between Perceived Risk, Evaluation, Satisfaction, and Behavioral Intention: A Case of Local-Festival Visitors. Journal of Travel \& Tourism Marketing. 33(1): 28-45.

SPSS, Statistical Package for the Social Sciences (2016). Versión 24. [En línea]. Disponible en: https://www.ibm.com/ mx-es/analytics/spss-statistics-software. Fecha de consulta: 14 de abril de 2020.

Stone, M. (1974). Cross-validatory choice and assessment of statistical predictions. Journal of the Royal Statistical Society. 36(2): 111-147.

UNESCO, Organización de las Naciones Unidas para la Educación, la Ciencia y la Cultura (2013). Creative Economy
Report 2013 Special Edition. [En línea]. Disponible en: https: //en.unesco.org/creativity/publication/creative-economyreport-2013. Fecha de consulta: 12 de octubre de 2020.

Yeoman, I., Robertson, M., Ali-Knight, J., Drummond, S., and McMahon-Beattie, U. (2004). Introduction. In I. Yeoman, M. Robertson, J. Ali-Knight, S. Drummond, and U. McMahon-Beattie (Eds.), Festival and Events Management (pp. 19-21). Oxford: Butterworth-Heinemann.

Zhang, C. X., Fong, L. H. N., and Li, S. (2019). Co-creation experience and place attachment: Festival evaluation. International Journal of Hospitality Management. 81: 193-204.

Ziakas, V. and Getz, D. (2021). Event portfolio management: An emerging transdisciplinary field of theory and praxis. Tourism Management. 83: 104233.

Zukin, S. (1995). The Cultures of Cities (First edition). Malden, MA: Blackwell. 336 Pp. 